\title{
North Carolina Appropriations for State-Supported College and University Libraries, I947`49
}

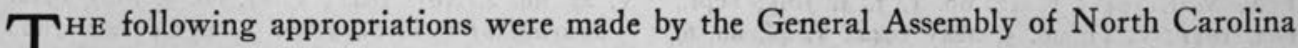
1 for the maintenance, buildings, and equipment of the libraries of state institutions for the biennium, 1947-49.

\section{Library}

University of North Carolina

(Library \$234,917)

(Law School 4,000)

University of North Carolina State College of Agriculture and

Engineering

University of North Carolina Woman's College

East Carolina Teachers College

Agricultural and Technical College (Negro)

Western Carolina Teachers College

Appalachian State Teachers College

Pembroke State College for Indians

Winston-Salem Teachers College (Negro)

Elizabeth City State Teachers College (Negro)

Fayetteville State Teachers College (Negro)

North Carolina College for Negroes North Carolina School for the Blind and Deaf

$$
\text { Total }
$$

\begin{tabular}{|c|c|c|c|c|}
\hline${ }_{1947-48}^{T o t a l}$ & $\begin{array}{l}\text { Books } \\
1947-48\end{array}$ & $\begin{array}{c}\text { Total } \\
1948-49\end{array}$ & $\begin{array}{l}\text { Books } \\
1948-49\end{array}$ & $\begin{array}{l}\text { Books- } \\
\text { Per Cent } \\
\text { of Total }\end{array}$ \\
\hline$\$ 238,917$ & $\$ 68,000$ & $\$ 238,917$ & $\$ 68,000$ & 28.46 \\
\hline $6 \mathrm{r}, 408$ & 25,000 & 62,008 & 25,000 & 40.31 \\
\hline 58,902 & 20,000 & $5^{8,902}$ & $20,000^{\prime}$ & 33.95 \\
\hline 21,780 & 8,500 & 1,780 & 8,500 & 39.04 \\
\hline 19,000 & 8,000 & 26,000 & $1_{5}, 000$ & 57.69 \\
\hline 30,300 & 5,000 & 30,300 & 5,000 & I 6.56 \\
\hline 22,980 & 7,000 & 22,980 & $7, \infty 00$ & 30.88 \\
\hline 5,690 & $4, \infty 00$ & 5,690 & $4, \infty 00$ & 70.28 \\
\hline $1_{3}, 600$ & 5,000 & $1_{3}, 600$ & 5,000 & 36.76 \\
\hline 10,330 & 4,000 & 10, 830 & 4,500 & 41.55 \\
\hline 10,040 & 4,500 & 10,140 & 4,500 & $44 \cdot 37$ \\
\hline 20,600 & 8,000 & 20,600 & 8,000 & 33.98 \\
\hline 830 & $\begin{array}{l}\left(\begin{array}{ll}S & 80\end{array}\right) \\
\left(\begin{array}{l}E \\
\text { 750 }\end{array}\right)\end{array}$ & $\$_{30}$ & $\begin{array}{l}\left(\begin{array}{ll}S & 80\end{array}\right) \\
\left(\begin{array}{l}E \\
\text { } 750\end{array}\right)\end{array}$ & \\
\hline 10,67 & $\$ 163,000$ & $\$ 518,877$ & $\$ 170,500$ & 32.85 \\
\hline
\end{tabular}

\section{BUILDINGS AND EQUIPMENT}

\section{Institution}

University of North Carolina

University of North Carolina Woman's College

East Carolina Teachers College

Agricultural and Technical College (Negro)

Western Carolina Teachers College

Fayetteville State Teachers College (Negro)

North Carolina School for the Blind and Deaf

Appalachian State Teachers College

Pembroke State College for Indians

North Carolina College for Negroes

\section{Total}

\begin{tabular}{rr} 
Equipment & Total \\
$\$ 75,000$ & $\$ 1,215,000$ \\
75,000 & 775,000 \\
40,200 & 446,000 \\
50,000 & 450,000 \\
40,000 & 304,800 \\
45,000 & 78,334 \\
7,000 & 108,000 \\
& 200,000 \\
50,000 & 200,000 \\
\hline$\$ 382,200$ & 550,000 \\
\hline & $\$ 4,327,134$
\end{tabular}

